\title{
HETERORREPARACIONES Y COMPETENCIA INTERACCIONAL DEL AULA: EL PAPEL DEL PROFESOR EN LA GESTIÓN DE LA INTERACCIÓN
}

\author{
Recepción: 30/10/2017 | Revisión: 31/12/2017 | Aceptación: 12/02/2018
}

\section{Jaume BATLLE RODRÍGUEZ}

Universitat de Barcelona

jaumebatlle@ub.edu

\begin{abstract}
Resumen: En el aula de lenguas extranjeras, las interacciones entre profesores y alumnos adquieren una especial relevancia, puesto que con ellas se gestiona y se produce aprendizaje. Bajo estos parámetros, profesores y alumnos deben ser capaces de llevar a cabo las interacciones de la mejor manera posible, en lo que se conoce como Competencia Interaccional del Aula. La reparación, como mecanismo para resolver problemas en interacción, es una de sus características. Así, si las interacciones entre profesores y alumnos están centradas en el significado, se entiende que las reparaciones deberán gestionarse para potenciar lo máximo posible la participación de los alumnos. Este artículo analiza cómo se gestionan las heterorreparaciones iniciadas por los alumnos en interacciones entre profesores y alumnos centradas en el significado. Específicamente, nuestra atención se focaliza en cómo la gestión repercute en la responsabilidad de restablecer la interacción tras la reparación. Del análisis se desprende que no siempre el restablecimiento de la interacción deriva de la propia alternancia de turnos de habla, sino que hay elementos en la gestión de la reparación que determinan quién vuelve al momento interaccional en que se marcó la existencia de un problema.
\end{abstract}

Palabras clave: interacción profesor-alumnos; competencia interaccional del aula; reparación; heterorreparación; análisis de la conversación.

\section{OTHER-REPAIR AND CLASSROOM INTERACTIONAL COMPETENCE:THETEACHER'S ROLE INTHE MANAGEMENT OF INTERACTION}

\begin{abstract}
In foreign language classroom, interactions between teachers and students are of great importance, as learning is operated and produced in interaction. Following these parameters, teachers and students must be able to carry out interactions in the best possible way, in what is known as Classroom Interactional Competence. Repair, as a mechanism to solve interactional problems, is one of its characteristics. Thus, if the teacher-students interactions are focused on meaning, repair must be managed to enhance students' participation as much as possible. This paper analyses how students-initiated other-repairs are managed in meaning-focused teacher-students interactions. Specifically, our aim is to observe the effect of interactional management on restoring interaction after a repair. Our data show that returning to the interaction not always comes from the turn-taking alternatives. On the contrary, there are features in the repair work that determine who comes back to the turn that was a trouble source.
\end{abstract}

Keywords: teacher-students interaction; classroom interactional competence; repair; other-repair; conversation analysis.

\section{HETERORREPARACIONS I COMPETĖNCIA INTERACCIONAL DE L'AULA: EL PAPER DEL PROFESSOR EN LA GESTIÓ DE LA INTERACCIÓ}

Resum: A l'aula de llengües estrangeres, les interaccions entre professors $i$ alumnes adquireixen una especial rellevància, ja que amb elles es gestiona $i$ es produeix aprenentatge. Seguint aquests paràmetres, professors $i$ alumnes han de ser capaços de dur a terme les interaccions de la millor manera possible, en el que es coneix com Competència Interaccional de l'Aula. La reparació, com a mecanisme per a resoldre problemes en interacció, és una de les seves característiques. Així doncs, si les interaccions entre professors $i$ alumnes estan centrades en el significat, s'entén que les reparacions s'hauran de gestionar per a potenciar el màxim possible la participació dels alumnes. Aquest article analitza com es gestionen les heterorreparacions iniciades pels alumnes en interaccions entre professors $i$ alumnes centrades en el significat. Especificament, centrem la nostra atenció en com la gestió repercuteix en la responsabilitat de restablir la interacció després de la reparació. De l’anàlisi es desprèn que no sempre el restabliment de la interacció deriva de la pròpia alternança de torns de parla, sinó que hi ha elements en la gestió de la reparació que determinen qui torna al moment interaccional en què es va marcar l'existència d'un problema.

Paraules clau: interacció professor-alumnes; competència interaccional de l'aula; reparació; heterorreparació; anàlisi de la conversa. 


\section{Introducción}

El aula de lenguas extranjeras es un espacio comunicativo en el que la interacción adquiere una relevancia especial: es la habilidad a partir de la cual se gestiona el aprendizaje. El docente, por norma general, decide quién participa y, dado que los turnos son contingentes entre sí, determina qué características presenta el turno de habla de su interlocutor. En consecuencia, la participación de los alumnos en la interacción normalmente está supeditada a la participación del profesor ${ }^{1}$. Asimismo, la participación del profesor puede determinar una mayor o menor efectividad en el aprendizaje de la lengua, puesto que no solo es que a través de la interacción se gestione el proceso de enseñanza-aprendizaje sino que, además, es posible considerar las interacciones que lleven a cabo los docentes con sus alumnos como oportunidades para el aprendizaje de la lengua meta.

La importancia de saber interactuar en el aula para los docentes cristaliza en el constructo de competencia interaccional del aula (Walsh, 2006, 2011), a partir del cual se pueden determinar prácticas interaccionales características del docente que le permiten buscar la eficiencia en su actividad. Tal caracterización supone entender que el profesor mantiene una serie de obligaciones interaccionales. En otras palabras, su rol social establece que su participación conlleve tener la responsabilidad de realizar determinadas prácticas interaccionales, en una búsqueda de una mayor efectividad de la interacción en relación con los objetivos de la misma. Así, la reparación de los problemas que emergen en la interacción es una tarea que los participantes en el acto de enseñanza-aprendizaje asumen como necesaria

El presente artículo indaga en la participación de los profesores de lenguas extranjeras en interacciones con los alumnos y centradas en el significado. Concretamente, nuestra atención va a focalizarse en cómo se gestiona la interacción en relación a la participación del profesor tras las reparaciones en las que el profesor solventa un problema ocurrido en la participación de los alumnos. Este tipo de reparaciones, conocidas como heterorreparaciones, implica que uno de los interlocutores, tanto el profesor como los alumnos, marcan la existencia de un problema en interacción y que este sea resuelto por el profesor.

\section{Marco teórico}

El Análisis de la Conversación como disciplina de estudio en el aula de lenguas extranjeras ha ha buscado comprender, entre otros aspectos, cómo las interacciones se desarrollan y articulan en este contexto específico. El patrón Iniciación-Respuesta-Feedback², comúnmente conocido por las siglas IRF (Sinclair y Coulthard, 1975; Mchoul, 1978; Mehan, 1979; Richards, 2006; Waring, 2008, 2009; Kääntä, 2010, entre otros) es el tipo de interacción preponderante en las aulas de lenguas extranjeras. La "arquitectura interaccional" (Seedhouse, 2004) de la clase de idiomas se fundamenta en este patrón interaccional. Según Seedhouse (2004), cuatro grandes tipos de interacción se producen en el aula de lenguas extranjeras: las interacciones centradas en la forma, las que están centradas en el contenido, las que están centradas en la tarea y las de carácter institucional. Cada una de ellas presenta una serie de características que las diferencian y son conformadas en función

1 A lo largo de todo el artículo, se utilizarán los términos professor y alumno como género no marcado.

2 También conocida como Iniciación-Respuesta-Evaluación o Iniciación-Respuesta-Follow up (Richards, 2006). 
de los objetivos pedagógicos que subyacen en ellas. El hecho de ser capaces de manejarlas de la manera más efectiva posible se considera fundamental para desarrollar lo que se conoce como competencia interaccional del aula (Walsh, 2006, 2011), concepto que se detallará a continuación.

\subsection{Competencia interaccional del aula}

La competencia interaccional del aula (CIA, en adelante) (Walsh, 2006, 2011; Sert, 2015; Hosoda y Aline, 2012; Escobar y Evnitskaya, 2014, Batlle, 2015; Acosta, 2017, entre otros) es un concepto que intenta definir aquellas habilidades que los participantes en el acto de enseñanza-aprendizaje presentan para llevar a cabo interacciones efectivas en función del objetivo de aprendizaje para las cuales hayan sido realizadas. El concepto deriva de la idea de competencia interaccional (Kramsch, 1986; Young, 2011; Hall, Hellermann y Pekarek Doehler, 2011, entre otros), comprendida esta como "the context-specific constellations of expectations and dispositions about our social worlds that we draw on to navigate our way through our interactions with others" (Hall y Pekarek Doehler, 2011: 1-2). En el habla en interacción, los hablantes son capaces de llevar a cabo acciones a partir de modelos determinados por el contexto en el que se producen las interacciones. Estas acciones determinan el desarrollo de la toma de turnos de habla, la organización de las acciones, y la gestión y monitorización de las interacciones momento a momento en función de las expectativas, recursos y orientaciones que los hablantes presentan. En el aula de lenguas extranjeras, la competencia interaccional adquiere una especial relevancia, puesto que a través de la interacción, como se apuntaba anteriormente, se gestiona y se genera el aprendizaje de la lengua meta. Específicamente, pues, el dominio de las interacciones propias del aula como microcosmos específico da pie a que se pueda considerar una competencia interaccional especializada: la CIA es aquella a partir de la cual los participantes desarrollan y ejecutan las acciones de enseñanza-aprendizaje.

Concretamente, por CIA se entiende la "teachers' and learners' ability to use interaction as a tool for mediating and assisting learning" (Walsh, 2006:130). La idea de CIA abarca las características de las interacciones del aula que hacen del proceso de enseñanza más o menos efectivo, tales como maximizar el espacio interaccional para que los estudiantes participen lo máximo posible, hacer un uso efectivo de la elicitación, ofrecer modelos de lengua u ofrecer reparación ante los problemas que puedan emerger en la interacción, entre otros aspectos (Sert, 2015:54). Las acciones que los profesores llevan a cabo en interacción con sus alumnos tienen repercusiones para el logro de los objetivos pedagógicos. Como destaca Steve Walsh (2013:55), las actuaciones de los profesores dan forma a las actuaciones de los alumnos y, en consecuencia, determinan la participación de los alumnos en la interacción. La relación de contingencia propia de toda interacción (Lee, 2007; Curl y Drew, 2008) y la progresividad inherente a ella (Heritage, 2007) implican que un turno de habla proyecte y moldee al siguiente (Liddicoat, 2004) y, por lo tanto, las características de un turno de habla determinan las del turno de habla posterior y vienen determinadas por las del turno de habla anterior. Por esta razón, podemos considerar que el profesor ocupa un papel central en las interacciones con los alumnos, puesto que su actuación determina la actuación del interlocutor.

En consecuencia, la CIA, como concepto que centra su atención en las interacciones que se generan en el aula, "focuses on the online decisions made by teachers and learners, and considers the extent to which these actions enhance learning and learning opportunities" (Seedhouse y Walsh, 
2010:139). Por ejemplo, si una interacción centrada en el significado está pensada para desarrollar en los alumnos la fluidez oral, el profesor debería permitir turnos de habla de los alumnos lo más largos posible, debería buscar que su participación fuera la mínima necesaria y debería minimizar las secuencias de reparación con el objetivo de que los estudiantes hablaran lo máximo posible (Walsh, 2011). Así, es posible determinar las repercusiones que determinadas acciones pueden tener para con el desarrollo de las interacciones y, por lo tanto, el aprendizaje de la lengua. Es el caso, como apuntábamos, de las secuencias de reparación

\subsection{Reparación y enseñanza de lenguas extranjera}

Una de las características inherente a toda interacción y a todo elemento involucrado en ella es que puede ser susceptible de considerarse como un problema. En tales casos, los hablantes hacemos uso de un dispositivo específico para solucionar lo antes posible el problema que haya emergido en interacción. Ese dispositivo se conoce como reparación (véase Schegloff, Sacks, y Jefferson, 1977; Hayashi, Raymond, y Sidnell, 2013; Batlle, 2015; Dingemanse et al., 2015).

Definida como "el tratamiento de un problema que ocurre en un uso interactivo de la lengua" (Batlle, 2015:118), la reparación puede ejecutarse por cualquier tipo de problema que ocurra en interacción, ya sea por un problema de audición, de comprensión o de expresión. La trayectoria reparacional se inicia justo en el momento en que un hablante considera algún elemento como problemático, marcándolo como reparable, e implica la existencia de un elemento posterior encaminado a solucionar el problema que se ha producido. Las reparaciones se ejecutan, pues, para minimizar en la medida de lo posible el problema y volver a la interacción en curso lo antes posible, restableciendo la intersubjetividad (Sidnell, 2015) inherente a toda interacción (Drew, 2005; Hayashi, 2005; Heritage, 2005, 2007).

Aunque la reparación "is a distinct practice from turn taking, it can only be understood in terms of turn taking and sequential position" (Markee, 2008:405), se puede analizar desde un punto de vista secuencial. Schegloff et al. (1977) determinaron cuatro tipos de reparaciones en función de quién marca la existencia del problema (quien lo ha producido o su interlocutor) y da solución al problema (quien lo ha producido o su interlocutor). Estos mismos autores determinaron que la reparación preferida en interacción es aquella en la que el mismo hablante que produce el problema lo soluciona, en lo que se conoce por autorreparación autoniciada. En cuanto antes se solucione el problema en cuestión, antes se volverá a la interacción en curso y, por lo tanto, la intersubjetividad inherente a la interacción quedará restablecida lo antes posible. Sin embargo, en un contexto pedagógico, autores como Kasper (1985) o Seedhouse (2004) determinan que las heterorreparaciones (HR), es decir, aquellas reparaciones en las que quien soluciona el problema no es el interlocutor que lo ha producido, son mucho más comunes. Se entiende, pues, que en el aula de lenguas extranjeras es común que los problemas que produzcan los estudiantes puedan ser reparados por el profesor.

\subsection{Las heterorreparaciones en las interacciones entre profesores y alumnos}

A Las HR se caracterizan, en el habla cotidiana, por ejecutarse en el turno de habla siguiente al que contiene el problema (Schegloff, 2000). Este tipo de reparación se asocia a contextos interacciona- 
les en los que la relación entre los interlocutores es asimétrica, como es el contexto pedagógico. En ese contexto, el tipo de HR más característico es la corrección, considerada desde la perspectiva del Análisis de la Conversación como un tipo específico de reparación (Batlle, 2015; Kendrick, 2015). La corrección es una HR caracterizada por el hecho de que el profesor marca la existencia del problema y aporta la solución en el mismo turno de habla y a través del mismo elemento. Sin embargo, en un contexto pedagógico también se pueden considerar HR aquellas secuencias cuyo problema está marcado por el alumno pero solucionado por el profesor.

Por su carácter educativo, la HR puede considerarse como " a device for dealing with those who are still learning or being taught to operate with a system which requires, for its routine operation, that they be adequate" (Forrester 2008:101). Por ejemplo, las reparaciones en las que el estudiante busca o desconoce una palabra y solicita ayuda a su interlocutor se podrían clasificar como HR (Mori y Hasegawa, 2009; Koshik y Seo, 2012), en este caso, HR autoiniciadas. Koshik y Seo (2012) indican que este tipo de reparaciones puede implicar una nueva secuencia de reparación si el elemento reparador que ofrece el profesor no se entiende como adecuado, aunque, por norma general, el profesor da muestras de escucha activa en señal de que el elemento utilizado por el alumno es adecuado e indicándole que puede continuar hablando. Sin embargo, si el profesor repite el elemento reparable o reformula el enunciado, corrigiéndolo, se estará aportando un elemento reparador.

En este tipo de reparaciones, emerge el estatus de los interlocutores y la posición epistémica de cada uno de ellos (Jakonen y Morton, 2015). El alumno presenta un dominio lingüístico que implica menos conocimiento en relación al uso de la lengua de comunicación y, en consecuencia, solicita ayuda al profesor, quien, por su estatus y su rol social en el contexto de interacción, se percibe como un interlocutor con un conocimiento epistémico superior en relación al uso de la lengua. Los alumnos, al iniciar la secuencia de reparación, buscan que el profesor les ayude a construir sus enunciados, por lo que se puede afirmar que, a través de estas secuencias de interacción, el aprendizaje "is located in a socially shared cognition as a practical accomplishment" (Kasper, 2009:32).

En línea con lo anterior, Koshik y Seo (2012) determinan que las estrategias comúnmente utilizadas por los alumnos para iniciar las HR autoiniciadas son aquellas que se construyen a partir de expresiones como “icómo puedo decir...?” o “¿cómo se dice...?” antes de producir el elemento reparable. En los ejemplos que nos proporcionan Koshik y Seo, se observa que, una vez el profesor ha ofrecido solución al problema, el alumno repite el elemento reparador, el profesor corrobora y, posteriormente, el alumno continúa con lo que estaba diciendo. Por lo tanto, es el alumno quien restablece la interacción truncada al iniciarse la secuencia de reparación.

Los estudios centrados en las heterorreparaciones en el aula de segundas lenguas han detallado sus características interaccionales: cómo se inicia la reparación y cómo se soluciona el problema han sido los principales focos de atención investigativa. Sin embargo, para determinar la competencia interaccional del aula implicada en el uso de las HR, se debe tener también en cuenta qué es lo que ocurre tras la secuencia de reparación. En este sentido, el hecho de continuar con la interacción en curso, en interacciones centradas en el significado, puede determinar una mayor o menor participación de los alumnos y, por lo tanto, puede ser una acción que se entienda dentro de los parámetros de la competencia interaccional del aula. Este estudio está encaminado a conocer qué es lo que ocurre tras tales secuencias de interacción para determinar qué implicaciones 
tiene la participación del profesor y las características de sus turnos de habla a la hora de determinar quién restablece la interacción tras la secuencia de reparación.

\section{Objetivos}

El análisis de las interacciones en el aula de español como lengua extranjera surge de la necesidad de conocer cómo se gestiona la participación de tanto de alumnos como de profesores para, a partir del conocimiento que surge, poder establecer acciones interaccionales que mejoren las acciones de profesores y alumnos y permitan un aprendizaje más efectivo de la lengua. En este sentido, la CIA permite establecer patrones que mejoran las acciones en interacción y hacen más efectiva la actuación de los participantes en el proceso de enseñanza-aprendizaje. Específicamente, en interacciones centradas en el significado, la posibilidad de restablecer la interacción por parte de los interlocutores tras una secuencia de reparación puede tener implicaciones en el desarrollo de la CIA, puesto que, entre otros factores, determina la posibilidad de que el estudiante continúe hablando y pueda participar lo máximo posible. Esta acción, si es llevada a cabo por el alumno, puede implicar que la interacción siga su curso y que este pueda continuar hablando. En cambio, si es llevada a cabo por el profesor, puede implicar que el alumno deje de hablar o no pueda desarrollar el enunciado en curso, el enunciado en el que este ha marcado la existencia de un problema.

En consecuencia, el objetivo de este estudio es analizar cómo se restablece la interacción tras la gestión de un tipo de secuencias de reparación específicas, aquellas HR iniciadas por los alumnos y resueltas por el profesor. En concreto, vamos a fijarnos en quién es el encargado de restablecer la interacción y qué relación tiene la potestad de volver a la interacción previa a la secuencia de reparación y cuáles son las acciones interaccionales implicadas en que sea uno u otro quien realice tal acción. Con ello, pretendemos ofrecer una visión precisa acerca de un aspecto propio de la gestión de problemas en interacción en el aula de español como lengua extranjera para que los lectores puedan aplicar tal conocimiento en su práctica docente habitual.

\section{Metodología}

El AC es una disciplina que busca comprender cómo se establecen las relaciones sociales a partir de las prácticas interaccionales. Se busca observar la organización de la interacción y los mecanismos implicados en ella para mostrar "how those practices are constructed by and for the participants in the interaction" (Brouwer y Wagner, 2004:32). En este sentido, "it is not just a matter of understanding the propositional content of what $T$ says in the L2; it is also a matter of analysing what social and sequential action $T$ is performing and what an appropriate social and sequential action in response would be" (Seedhouse, 2005:177). En consecuencia, se adopta una perspectiva émica con la intención de identificar modelos de acción social y reconocer acciones significativas en una relación reflexiva, entre las acciones analizadas y su interpretación, según la cual "the same set of methods or procedures are responsible for both the production of actions/utterances and their interpretation" (Seedhouse, 2004:11).

Las interacciones analizadas siguen el modelo denominado Round Robin (Mortensen y Hazel, 2011). Son interacciones centradas en el significado que se pueden clasificar como Classroom Context Mode, según la tipología de interacciones del aula que determina Walsh (2006). En 
concreto, son interacciones centradas en el significado (Seedhouse, 2004) en las que el profesor va preguntando a los alumnos, uno a uno, por lo que hicieron durante el fin de semana anterior a la sesión de clase o por lo que harán durante el fin de semana siguiente a la clase. Todas las interacciones se generaron al iniciar la sesión, por lo que se pueden catalogar, asimismo, como discurso social (González Argüello, 2010). El grupo clase estaba conformado por doce alumnos de nivel A1+ de diversas procedencias, mayoritariamente estadounidenses, que habían venido a Barcelona a cursar un semestre dentro de un programa de intercambio universitario: los estudiantes norteamericanos cursaban un semestre dentro de un programa de Study Abroad, mientras que el resto eran estudiantes dentro del programa Erasmus. Las interacciones objeto de estudio forman parte de un corpus de datos de 40 horas de grabaciones de clase realizadas durante un curso extensivo de español. En las sesiones grabadas, un total de 8 interacciones, con un total de 1 hora y 33 minutos, cumplieron con las características especificadas anteriormente. Los datos han sido transcritos siguiendo el sistema propio del análisis de la conversación (véase Jefferson, 2004; Hepburn y Bolden, 2017).

\section{Análisis de datos y resultados}

Como se ha comentado anteriormente, la reparación es un mecanismo interaccional que implica la momentánea ruptura en la intersubjetividad inherente a la interacción para solucionar un problema que ha emergido. En las interacciones entre profesores y alumnos centradas en el significado en las que los profesores preguntan a los alumnos por información y estos responden, suele ser habitual que los alumnos tengan algún tipo de dificultad para expresar sus ideas, por lo que solicitan ayuda al profesor para que, dado su conocimiento lingüístico, pueda solventar el problema que presentan. Las reparaciones iniciadas por los alumnos y cuyo problema es solventado por el profesor son un tipo clásico de esta organización secuencial.

Puesto que la secuencia de reparación detiene momentáneamente la acción en curso, la recuperación de la interacción en el punto en que se encontraba anteriormente resulta una acción significativa. Por norma general, la alternancia de turnos de habla inherente a toda interacción puede determinar que, si el profesor es quien lleva a cabo la reparación, sea el alumno el que asuma la responsabilidad de continuar con la interacción. El alumno, en el siguiente turno de habla, posterior al turno reparador, puede recurrir al elemento que el profesor le ha ofrecido y reformular el turno de habla que contenía el problema para expresar lo que quería decir. Es el caso que se observa en (1), en el que el alumno inicia una secuencia de reparación al no saber si la palabra intensivo (línea 2) es adecuada en español.

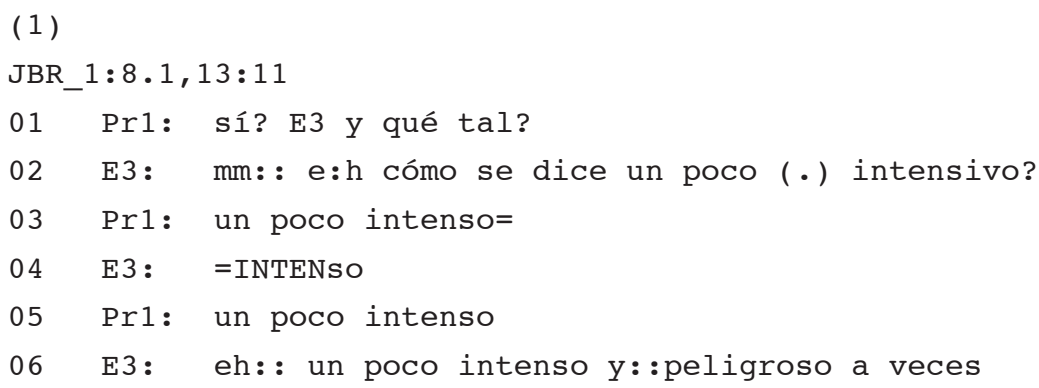


El alumno inicia la secuencia de reparación a partir del uso de una pregunta. Con "cómo se dice" está considerando el elemento con el que construye su turno de habla como problemático. La pausa posterior a "un poco" y la entonación ascendente determina que el elemento problemático es el que se formula en última posición. El profesor, para reparar el problema del alumno, reconstruye el enunciado y lo reformula, incorporando la solución léxica que considera adecuada en función de lo que el alumno quiere decir. Ante esta acción, el alumno recoge el elemento reparador que le ha ofrecido el profesor y formula otra vez el enunciado, esta vez continuando con la explicación de cómo le fue el fin de semana. Se observa, pues, que la continuación de la interacción puede correr a cargo del alumno si el restablecimiento de la interacción se produce en el turno de habla justamente posterior a la formulación del elemento reparador por parte del profesor. En este caso, la secuencia interaccional no se ve alterada y se vuelve a la interacción con la alternancia de turnos propia de la secuencia.

Sin embargo, en algunas ocasiones, la secuencia de reparación puede llevarse a cabo de una manera más compleja. A veces, la solución del profesor al problema acarrea la necesidad de ser reparada, por lo que dentro de la secuencia de reparación se genera otra secuencia de reparación. La complejidad secuencial de este tipo de secuencias implica que para solucionar el problema iniciado por el alumno se deba, anteriormente, cerrar la secuencia de reparación iniciada posteriormente. En otras palabras, en secuencias de reparación complejas, como la que se observa en (2), es necesario solucionar, en primer lugar, el problema ocurrido en segundo lugar.

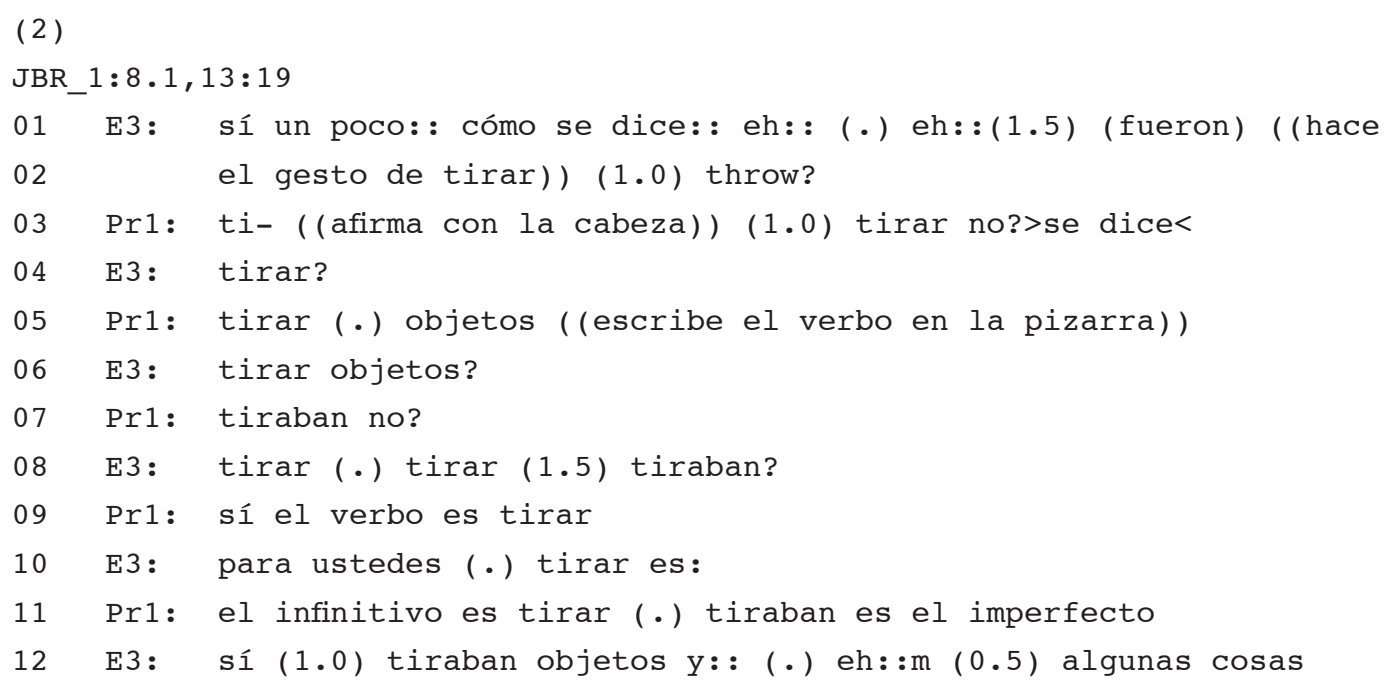

La reparación por parte del alumno se marca con dos diferentes iniciadores. En una primera instancia, el alumno, justo en el momento en que percibe un problema en su conocimiento lingüístico, lleva a cabo una iniciación de reparación a partir del enunciado "cómo se dice" (línea 01), el cual viene acompañado por algunas pausas oralizadas, señal de que está pensando en qué estrategia usar para determinar el problema y que, a partir de ahí, el profesor pueda repararlo. El problema queda determinado a partir de dos recursos: el uso de la palabra que quiere decir, pero en su versión en inglés, y un gesto con el que se puede identificar lo que quiere decir. El profesor, en ese momento, identifica el problema y ofrece una reparación al alumno. En la línea 2, este indi- 
ca que el verbo que tenía que utilizar el alumno era 'tirar'. Sin embargo, E3 inicia una secuencia de reparación en la búsqueda de corroboración de que la reparación que ha proporcionado el profesor es adecuada (líneas 05 y 07). Ante ello, su interlocutor, el profesor, corrobora que el elemento reparador es adecuado para construir el enunciado que el alumno quiere construir, pero, como se observa en la línea 07 , reformula el elemento reparador al conjugar el verbo. En ese momento, está introduciendo una nueva variable a partir de la cual el alumno puede construir con más precisión la acción que pretendía llevar a cabo. Ello produce que el alumno inicie una nueva secuencia de reparación similar a la anterior puesto que marca el elemento "tiraban" (línea 08) como un elemento que necesita corroborar. El profesor, ante tal acción, aporta información de carácter metalingüístico, centrándose en las características formales del elemento reparador: indica que 'tiraban' es la forma de imperfecto del verbo 'tirar' que el alumno puede utilizar.

La solución de estas secuencias intercaladas dentro de la primera secuencia de reparación implica que el alumno ya se vea en la tesitura de utilizar el elemento reparador y reformular el enunciado inicial, en el que había marcado la existencia del problema. Sin embargo, antes de reiniciar su enunciado, lleva a cabo una afirmación. Con este inicio de turno de habla, E3 indica que confirma la adecuación del turno de habla anterior. Hay que tener en cuenta que tal afirmación no se produce por el hecho de que el profesor le haya preguntado por la adecuación del elemento problemático, que, como se verá más adelante, tiene unas diferentes repercusiones interaccionales, sino que, con 'sí' (línea 12), simplemente reafirma la adecuación de la reparación proporcionada por el profesor.

La continuación de la interacción tras la secuencia de reparación que se acaba de analizar corre a cargo del alumno. Se observa, pues, que la responsabilidad de restablecer con la interacción, en el caso de que se produzcan secuencias de reparación insertas en otras secuencias, puede caer en el alumno, siguiendo, así, la distribución de la toma de turnos prototípica de la interacción. Debemos tener en cuenta que el profesor formula el turno de habla reparador únicamente con la intención de proporcionar ese elemento que solicita el alumno, a pesar de las reparaciones insertas que se encuentran dentro de la secuencia de reparación. En este sentido, en los dos extractos que acabamos de analizar, la acción que lleva a cabo el profesor únicamente está pensada para ofrecer al alumno el elemento a partir del cual se puede solucionar el problema cuya existencia ha marcado. Sin embargo, la participación del profesor en las secuencias de reparación iniciadas por los alumnos no tiene por qué reducirse a un mero ofrecimiento del elemento reparador, sino que puede conllevar otras acciones.

En otros momentos, la acción reparadora debe consolidarse con algún tipo de explicación que dé fuerza a la solución que se acaba de proporcionar. Esta explicación es ofrecida por el profesor ante solicitudes de corroboración por parte del alumno. En ocasiones, la solución del profesor no es comprendida por el alumno o, en su defecto, necesita ser corroborada. En (3), por ejemplo, el alumno infiere que el verbo que debe utilizar para construir su enunciado es 'gastar' (línea 1), pero no está seguro de ello. En consecuencia, le pide ayuda al profesor iniciando una secuencia de reparación. 
( 3 )

JBR_1:6.1, $14: 23$

01 E3: [sí sí (.) y eh: (.) yo quiero: gastar? (0.5) eh: :mhm cómo

02 se dice: (.) mucho tiempo?

03 Pr1: pasar se dice

04 E3: pasar?=

$05 \operatorname{Pr} 1:=$ spend time=

06 E3: =sí

07 Pr1: es pasar tiempo ono eso gastar en español es so[lo para el=

08 E3: [solo dinero

09 Pr1: =dinero [vale?]

10 E3: [ sí ] (.) sí sí (.) eh: : porque tengo un amigo

El enunciado que construye el alumno le proporciona información al profesor sobre qué verbo debería haber utilizado su interlocutor. Así, el docente puede ofrecer un elemento reparador con seguridad. Sin embargo, el uso del verbo pasar en ese contexto es desconocido para el alumno, por lo que inicia otra secuencia de reparación (línea 4) con la que pretende que el profesor le explique por qué se debe usar pasar ahí. El profesor traduce el término al inglés, acción que es corroborada por el alumno (línea 06). El profesor, conocedor de que el verbo 'spend' en inglés puede traducirse de dos maneras diferentes en función del contexto de uso, ofrece a su interlocutor una explicación sobre cuándo se utiliza gastar y cuándo se utiliza pasar.

El restablecimiento de la interacción tras esta secuencia de reparación corre a cargo del alumno. Debemos tener en cuenta que, a pesar de que el profesor formula una coda al final de su turno de habla que podría implicar únicamente una afirmación por parte del alumno (véase el análisis del siguiente extracto), esta acción comunicativa se lleva a cabo de forma solapada. Además, el alumno previamente ya había intentado ganar la posibilidad de hablar con otro inicio de turno de habla solapado (línea 08), por lo que se deduce que quiere participar en la interacción para hablar de su experiencia. La participación del alumno no se reduce únicamente a corroborar la apreciación del profesor y, por lo tanto, a validar la reparación proporcionada, sino que sus acciones muestran una clara voluntad de querer seguir participando. Los solapamientos nos dan a entender que el alumno tiene la pretensión de continuar hablando a pesar de que podría producir un turno de habla para únicamente afirmar la adecuación de la reparación. Este hecho es significativo para entender por qué E3 es el encargado de restablecer la interacción tras la secuencia de reparación.

En los ejemplos (2) y (3), se ha observado que el profesor interviene cuando el alumno inicia una secuencia de reparación marcando como problemático el elemento que el profesor ha propuesto en primera instancia como solución al problema anterior. y que el alumno puede corroborar o no la adecuación del elemento reparador. El diseño de la acción corroborativa tiene repercusiones interaccionales. Mientras que en caso de (2) el alumno corroboraba la acción reparadora con una afirmación y continuaba con la acción en curso -en consecuencia, era él quien tenía la potestad de restablecer la interacción tras la secuencia de reparación-, el hecho de que el alumno indique que la reparación es adecuada a partir de una señal de cambio de estado implica que su turno de habla quede reducido a esa acción únicamente. En las líneas 14 y 16 del extracto 
(4), el alumno asume la adecuación de la reparación a partir de "ah", elemento que indica que el alumno reformula su conocimiento epistémico con respecto a algún elemento del enunciado anterior. En este caso, las señales de cambio de estado determinan que E3 no conocía el significado del verbo 'llevar', elemento reparador que utiliza el profesor y con el que soluciona la secuencia de reparación que el alumno había iniciado. Cabe destacar, sin embargo, que el desconocimiento del elemento reparador por parte del alumno se hace explícito en una segunda iniciación de reparación por parte del alumno, precisamente con la intención de entender qué significa la palabra que el profesor ha utilizado como elemento reparador para el primer problema (línea 9).

(4)

JBR_ $1: 1.1,8: 48$

01 E3: [un poco] ( (entra una alumno: es E4, que siempre llega un poco

02 tarde)) un poco más que eh:: aquí em: : pero eh:: he traído? I

03 brought?

04 Pr1: sí

05 E3: mi chaqueta? ((gestos de afirmación del profesor con la cabeza))

06 y mi chaqueta para llovar? [( )]

07 Pr1: [mhm para la] lluvia

08 E3: [ $\left(\begin{array}{ll})\end{array}\right]$

09 Pr1: [he tra]ído no, he llevado ((acompaña con gestos))

10 E3: $\mathrm{e}-=$

11 Pr1: =vale? he llevado

12 E3: he llevado? qué es?

13 Pr1: up- (.) to bear ((gestos))

14 E3: ah: : :

15 Prl: no? pues he traído a Barcelona, es he llevado a Budapest=

16 E3: =ah: :

17 Pr1: vale? la chaqueta

18 E3: sí sí

19 Pr1: y con eso bien

20 E3: cómo?

21 Pr1: con la chaqueta bien

22 E3: sí sí

$23(1.0)$

24 Pr1: E10, veo que tienes muchas ganas de hablar, qué has hecho este 25 fin de semana?

El hecho de que el profesor buque la corroboración del alumno con la pregunta "vale?" (línea 17) implica una respuesta afirmativa por parte del alumno. La acción de E3 se conforma, pues, en función del turno de habla anterior. Su foco de atención está en responder que valida la acción reparadora del profesor. A diferencia de la acción que se observaba en (3), en la que el alumno, de forma solapada, intentaba tomar el turno de habla, el alumno no continúa con la acción en curso previa a la secuencia de reparación. Es el profesor quien, recoge lo que estaba diciendo el alumno y produce un turno de habla con el que pretende que este último le diga que con la chaqueta no pasó 
frío en su visita a Budapest. En tal momento, se puede observar que es el profesor quien restablece la acción en curso y, por lo tanto, quien asume la responsabilidad de continuar con la interacción. No es el alumno quien continúa hablando de su viaje a Budapest, sino que es el profesor quien lo hace a partir de un turno de habla con el que pretende que E3 diga que la chaqueta le sirvió en su viaje. Posteriormente, tras la afirmación del alumno de que lo que dice el profesor es cierto y una pausa de un segundo, el profesor se ve con la potestad de cambiar de interlocutor y, por lo tanto, de propiciar la participación de otro alumno.

Como se observa, las reparaciones que implican que el alumno tenga que atestiguar que la reparación llevada a cabo por el profesor es adecuada tras una solicitud explícita por parte del profesor implican que sea este último quien tenga la responsabilidad de continuar con la acción en curso. En otras palabras, cuando el profesor explícitamente lleva a cabo una acción a partir de la cual el alumno tiene que responder "sí" o "no", el profesor está proyectando un turno de habla de su interlocutor centrado en la elaboración de la respuesta y no en la elaboración de un turno de habla relacionado con la expresión de la experiencia del alumno.

Las reparaciones en las que los alumnos solicitan la ayuda al profesor para lograr solventar un problema que presentan en la construcción de sus turnos de habla pueden ejecutarse a partir de propuestas en las que el profesor busca corroboración del elemento que proponen como reparador. En algunos casos, el profesor puede dudar en relación con la adecuación del elemento reparador, ante lo que forman una acción que busca que el interlocutor, quien ha solicitado ayuda, tenga que corroborar la adecuación del intento de reparación. En (5), vemos que E3 quiere expresar que, en su viaje a Ámsterdam, visitó la cervecería Heineken. Sin embargo, solicita la ayuda del profesor al no saber cómo se dice en español "los lugares para hacer cerveza". 
En una primera instancia, el profesor ofrece una primera solución al problema anterior con la incorporación del verbo 'fabricar' (línea 05), solución que es corroborada por el alumno. Por lo tanto, la acción del profesor proyecta un turno de habla por parte del alumno destinado a afirmar que la acción reparadora es adecuada. El alumno, a pesar de que podría tener la posibilidad de continuar con la acción en curso cortada por la secuencia de reparación, únicamente se centra en responder a la pregunta anterior. Tras la corroboración de la línea 06, el profesor intuye qué es lo que quería decir el alumno y, ante ello, procede a explicar cómo se expresa la idea que E3 quería comunicar. El profesor ofrece una solución con la palabra 'destilería' (línea 10), pero, además, intuye que la solución puede no ser oportuna, por lo que explica que, normalmente, destilería se utiliza más para el whisky. La explicación del vocablo, en este caso, ofrece la posibilidad al profesor de tomar el turno de habla y, en consecuencia, de reanudar la interacción y restablecer la intersubjetividad subyacente. La acción de la línea 16 está encaminada para ello: el profesor, al tener ganado el turno de habla, reanuda la interacción preguntándole al alumno qué más cosas hizo en su visita a Ámsterdam.

En el ejemplo anterior se observa que la reanudación de la interacción corre a cargo del profesor. El hecho de que el profesor haya proporcionado información sobre el elemento problemático da pie a que tenga tomado el turno de palabra y, en consecuencia, se vea con la potestad de reanudar la interacción. Así, se observa que el profesor puede ser el encargado de restablecer la interacción tras una secuencia de reparación si él mismo lleva a cabo una explicación del vocablo con el que intenta solventar el problema. Es el mismo caso que en (6), en el cual el profesor, directamente, soluciona el problema de carácter léxico que muestra el alumno y, a continuación, ofrece una explicación de qué significa 'disfrazarse' (líneas 03-06).

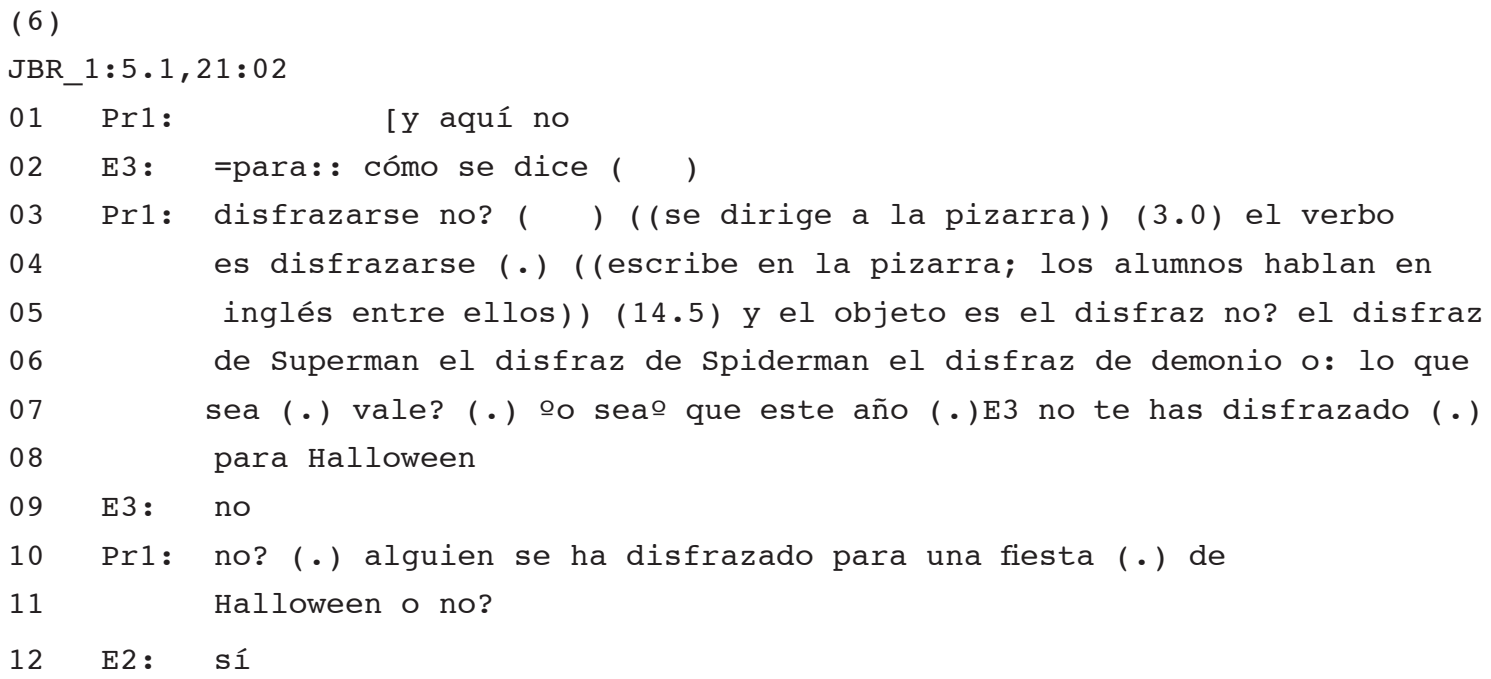

La explicación de qué significa disfrazarse se apoya en la descripción de lo que es un disfraz. Tras escribir el término en la pizarra, acción que implica que el resto de la clase focalice la atención en el elemento problemático, el docente restablece la interacción a partir de una reformulación de lo que el alumno estaba diciendo. Le pregunta si se había disfrazado, ante lo cual el alumno le responde negativamente. Esta acción implica que el turno de habla del alumno esté supeditado a 
la pregunta a la que responde. La pregunta formulada proyecta un turno de habla que se reduce únicamente a responder a la acción anterior. Por lo tanto, la formulación de la pregunta no propicia que el alumno siga hablando. En el siguiente turno de habla, el profesor cambia de interlocutor.

Dadas las relaciones de contingencia implicadas en las relaciones entre los turnos de habla, podemos atestiguar que las acciones implicadas en las secuencias de reparación por parte de los interlocutores son determinantes para restablecer la interacción que se estaba llevando a cabo y, por lo tanto, para saber qué interlocutor tiene la potestad de restablecer la interacción. La alternancia de turnos de habla propia de la interacción determina que, si el profesor ofrece la solución, el alumno, en el siguiente turno, pueda continuar con lo que estaba diciendo e incorporar el elemento reparador a su enunciado. Sin embargo, en ocasiones, las HR que se han analizado se gestionan más allá del siguiente turno, ante lo cual las acciones implicadas en la secuencia de reparación son significativas para obtener la posibilidad de restablecer la interacción anterior. Como muestran los datos, las HR en las que la solución al problema provoca la iniciación de una secuencia de reparación pueden implicar la posibilidad de que el profesor lleve a cabo algún tipo de explicación para consolidar el elemento reparador como válido. En tales casos, el profesor puede buscar la corroboración del alumno y, si este último no muestra una voluntad expresa de seguir con la interacción y únicamente formula turnos de habla afirmando la adecuación de la reparación, es el profesor el que tiene la potestad de restablecer la secuencia de interacción que había sido truncada por la explicitación de la existencia de un problema por parte del alumno. Por lo tanto, parece que, si la reparación que inicia el alumno al no saber cómo se dice algo en español se soluciona a partir de una reparación en el siguiente turno, el alumno tendrá la posibilidad de restablecer la interacción. Si, en cambio, la secuencia de reparación se vuelve compleja, habrá factores interaccionales relacionados con las relaciones entre turnos de habla, tales como la contingencia inherente entre turnos o la proyectabilidad que un primer turno establece sobre un segundo, que determinen qué interlocutor tendrá la potestad de restablecer la interacción tras la secuencia de reparación. Como se ha observado en los datos, si se llevan a cabo explicaciones de carácter metalingüístico, en nuestros casos, explicaciones normalmente que aclaran el término reparador, el profesor puede verse con la posibilidad de restablecer la interacción. Además, si el profesor busca la corroboración del alumno sobre la adecuación de la reparación, este último podrá centrar su acción en responder a tal corroboración, puesto que esta acción proyecta un turno de habla posterior que implica la afirmación o negación de lo dicho anteriormente. En tales casos, el alumno está formando una acción centrada en responder a la acción anterior y no en continuar con lo que estaba diciendo antes de la iniciación de la secuencia de reparación.

\section{Conclusiones}

El análisis llevado a cabo en este estudio ha podido determinar que la continuación de la interacción tras HR llevadas a cabo por el profesor y en las que los alumnos indican la existencia de un problema para la producción de sus enunciados puede ir a cargo tanto del profesor como de los alumnos. Si la reparación es en el siguiente turno y el elemento reparador no implica la iniciación de otra secuencia de reparación, la alternancia de turnos de habla implica que sea el alumno el que continúe con la interacción y, en consecuencia, pueda seguir hablando y desarrollando las ideas 
que estaba expresando antes de marcar la existencia del problema. Por el contrario, si la secuencia de reparación se lleva a cabo en una secuencia de más de dos turnos de habla, el alumno podrá restablecer la interacción siempre y cuando se creen las condiciones interaccionales para ello. Tales casos ocurren cuando hay una voluntad explícita por parte del alumno de querer seguir hablando o cuando no genera turnos de habla reparadores. En el caso de que el profesor aporte explicaciones relacionadas con el elemento reparador puede verse con la potestad de restablecer él la interacción o de solicitar al alumno que corrobore que la solución aportada es la adecuada. En este último caso, dada la contingencia propia de la interacción, el alumno puede focalizar su atención únicamente en la corroboración y, en consecuencia, perderá la posibilidad de recuperar aquello que estaba intentando decir en el enunciado en el que formula la existencia del problema inicial.

Las caracterizaciones mostradas en el análisis anterior muestran la complejidad de un fenómeno interaccional aparentemente simple: el restablecimiento de la interacción tras una HR producida por el profesor. Sin embargo, el hecho de que sea el profesor o el alumno quien restablezca la interacción caracteriza las acciones interaccionales que se llevan a cabo en el aula y, en consecuencia, tiene implicaciones para el desarrollo y la comprensión de la competencia interaccional del aula. Desde la perspectiva de la Competencia Interaccional del Aula, uno de los objetivos de las interacciones centradas en el significado está en que los alumnos puedan participar lo máximo posible y, por lo tanto, puedan mejorar en relación con el uso de la lengua en interacción. El hecho de que el alumno pueda continuar con la interacción en curso tras haber marcado la existencia de un problema da pie a ello. El alumno, cuando puede restablecer la interacción, restablece también la posibilidad de continuar participando y, en consecuencia, de ser protagonista y desarrollar una participación significativa que le reporte en una mejora en relación con el uso de la lengua. Por lo tanto, implica un mayor desarrollo de su competencia comunicativa. Al contrario, si el profesor es quien restablece la interacción, las posibilidades de que el alumno que está participando pueda continuar expresando lo que quería decir se reducen. Así, es conveniente que se tenga en cuenta que, en ocasiones, la solución de problemas por parte del profesor y cuya existencia es determinada por el alumno puede implicar que los alumnos no puedan continuar desarrollando sus ideas. El profesor, como gestor de la interacción, debe ser consciente de las consecuencias que pueden tener sus intervenciones en tales secuencias interaccionales. Los datos han mostrado que la solicitud de corroboración por parte del profesor puede implicar que el alumno focalice su atención en confirmar la adecuación de la solución, lo que da pie al profesor a continuar con la interacción y, si lo cree conveniente, a cambiar de interlocutor. En todo caso, no se le da la oportunidad al alumno de que continúe desarrollando sus ideas.

Se puede llegar a pensar que el alumno no continúa con el desarrollo de su experiencia porque no lo cree conveniente. Ahora bien, a pesar de que pueda ser así, como profesores, debemos siempre incitar a la participación y a que los alumnos sean protagonistas del aprendizaje que llevan a cabo. Este hecho implica que deban ser protagonistas en las interacciones que llevan a cabo con el profesor y, por tanto, que deban tener el máximo de posibilidades para participar. Esa es una de las premisas a partir de las cuales se puede decir que los participantes en la interacción son competentes desde la perspectiva de la CIA. Si, como profesores, somos más conscientes de cómo funciona el restablecimiento de las interacciones tras unas determinadas secuencias de re- 
paración, podremos llevar a cabo prácticas interaccionales más apropiadas para que los alumnos desarrollen su aprendizaje de la manera más efectiva posible.

\section{Referencias bibliográficas}

Acosta, L. (2017). La reparación en la interacción oral de estudiantes de ELE: comparación entre interacciones de práctica en el aula e interacciones en contextos de evaluación. Pragmática Sociocultural/Sociocultural Pragmatics, 5(2), 219-250.

Batlle, J. (2015). La organización secuencial de las reparaciones en interacciones entre profesor y alumnos de español como lengua extranjera centradas en el significado: Repercusión en la intersubjetividad y en la competencia interaccional de los hablantes. Tesis doctoral, Universitat de Barcelona. Disponible en: http://www.tdx.cat/handle/10803/390950

Brower, C., y Wagner, J. (2004). Developmental issues in second language conversation. Journal of Applied Linguistics, 1(1), 29-47.

Curl, T.S., y Drew, P. (2008). Contingency in action: A comparison of two forms of requesting. Research on Language and Social Interaction, 41(2), 129-153.

Dingemanse, M., Roberts, S., Baranova, J., Blythe, J., Drew, P., Floyd, S., Gisladottir, R., Kendrick, K., Levinson, S., Manrique, E., Rossi, G., y Enfield, N. (2015). Universal principles in the re-pair of communication problems. PLOS ONE, 10(9), e0136100. Recuperado de http:// journals.plos.org/plosone/article?id=10.1371/journal.pone.0136100

Drew, P. (2005). Conversation analysis. En K. Fitch y R. Sanders (Eds.), Handbook of language and social interaction, p. 71-102. NJ: Lawrence Erlbaum.

Escobar, C., y Evnitskaya, N. (2014). "Do you know Actimel?" The adaptive nature of dialogic teacher-led discussions in the CLIL science classroom: a case study. The Language Learning Journal, 42(2), 165-180.

Forrester, M.A. (2008). The emergence of self-repair: A case study of one child during the early preschool years. Research on Language and Social Interaction, 41(1), 99-128.

González Argüello, M.V. (2010). Análisis del discurso en el aula: una herramienta para nuestra autoevaluación. Marcoele, 10. Recuperado de http://marcoele.com/descargas/ expolin-gua_2006.gonzalez.pdf

Hall, J.K., Hellermann, J., y Pekarek-Doehler, S. (Eds.) (2011). L2 interactional competence and development. Bristol: Multilingual Matters.

Hayashi, M. (2005). Reference problems and turn construction: An exploration of an intersection between grammar and interaction. Text, 25, 437-468.

Hayashi, M., Raymond, G., y Sidnell, J. (Eds.) (2013). Conversational repair and human understanding. Cambridge: Cambridge University Press.

Hepburn, A., y Bolden. G. (2017). Transcribing for social research. London: SAGE.

Heritage, J. (2005). Conversation analysis and institutional talk. En K. Fitch y R. Sanders (Eds.), Handbook of language and social interaction, p. 103-137. NJ: Lawrence Erlbaum.

Heritage, J. (2007). Intersubjectivity and progressivity in person (and place) reference. En N. Einfield y T. Stivers (Eds.), Person and reference in interaction: Linguistic, cultural, and social perspectives, p. 255-280. Cambridge: Cambridge University Press.

Hosoda, Y., y Aline, D. (2012). Doing being interrupted by noise as a resource in second language interaction. Journal of Pragmatics, 44(1), 54-70.

Jakonen, T., y Morton, T. (2015). Epistemic search sequences in peer interaction in a contentbased language classroom. Applied Linguistics, 36(1), 73-94. 
Jefferson, G. (2004). Glossary of transcript symbols with an introduction. En G. Lerner (Ed.), Conversation analysis: Studies from the first generation, p. 13-31. Philadelphia: John Benjamins.

Kääntä (2010). Teacher turn-allocation and repair practices in classroom interaction: A multisemiotic perspective. Tesis doctoral. Universidad de Jyväskylä. Recuperado de https://jyx.jyu.fi/dspace/handle/123456789/22993

Kasper, G. (1985). Repair in foreign language teaching. Studies in Second Language Acquisition, 7, 200-215.

Kasper, G. (2009). Locating cognition in second language interactions and learning: inside the skull or in public view? International Review of Applied Linguistics in Language and Teaching, 47, 11-36.

Kendrick, K. (2015). The intersection of turn-taking and repair: the timing of other initiations of repair in conversation. Frontiers in Psychology, 6, 1-16. Recuperado de https://www.ncbi. nlm.nih.gov/pmc/articles/PMC4357221/

Koshik, I., y Seo, M. (2012). Word (and other) search sequences initiated by language learners. Text \& Talk, 32(2), 167-189.

Kramsch, C. (1986). From language proficiency to interactional competence. The Modern Language Journal, 70, 366-372.

Lee, Y. (2007). Third turn position in teacher talk: Contingency and the work of teaching. Journal of Pragmatics, 39(6), 1204-1230.

Liddicoat, A. (2004). The projectability of turn constructional units and the role of prediction in listening. Discourse Studies, 6(4), 449-471.

Markee, N. (2008). Toward a learning behaviour tracking methodology for CA-for-SLA. Applied Linguistics, 29(3), 404-427.

McHoul, A. (1978). The organization of turns at formal talk in the classroom. Language in Society, 7, 183-213.

Mehan, H. (1979). Learning lessons. Social organization in the classroom. Cambridge: Harvard University Press.

Mori, J., y Hasegawa, A. (2009). Doing being a foreign language learner in a classroom: Embodiment of cognitive states as social events. International Review of Applied Linguistics, 47(1), 65-94.

Mortensen, K, y Hazel, S. (2011). Initiating round robins in the L2 classroom - preliminary observations. NOVITAS-Royal, 5(1), 55-70. Recuperado de http://www.novitasroyal.org/ Vol_5_1/mortensen_hazel.pdf

Richards, K. (2006). 'Being the teacher': Identity and classroom conversation. Applied Linguistics, 27(1), 51-77.

Schegloff, E. (2000). When 'others' initiate repair. Applied Linguistics, 21(2), 205-243.

Schegloff, E., Sacks, H., y Jefferson, G. (1977). The preference for self-correction in the organization of repair in conversation. Language, 53, 361-382.

Seedhouse, P. (2004). The interactional architecture of language classroom: A conversation analysis perspective. Malden, MA.: Blackwell.

Seedhouse, P. (2005). Conversation analysis and language learning. Language Teaching, 38(4), 165-187.

Seedhouse, P., y Walsh, S. (2010). Learning a second language through classroom interaction. En P. Seedhouse, S. Walsh y C. Jenks (Eds.), Conceptualising learning in applied linguistics, p. 127-146. Basingstoke: Palgrave Macmillan.

Sert, O. (2015). Social interaction and L2 classroom discourse. Edinburgh: Edinburgh University Press. Sidnell, J. (2015). The architecture of intersubjectivity revisited. En N. Enfield, P. Kockelman y 
J. Sidnell (Eds.), The Cambridge handbook of linguistic anthropology, p. 364-399. Cambridge: Cambridge University Press.

Sinclair, J., y Coulthard, M. (1975). Towards an analysis of discourse. The English used by teachers and pupils. Oxford: Oxford University Press.

Walsh, S. (2006). Investigating classroom discourse. New York: Routledge.

Walsh, S. (2011). Exploring classroom discourse: Language in action. London: Routledge.

Walsh, S. (2013). Classroom discourse and teacher development. Edinburgh: Edinburgh University Press.

Waring, H.Z. (2008). Using explicit positive assessment in the language classroom: IRF, feedback, and learning opportunities. The Modern Language Journal, 92(4), 577-594.

Waring, H.Z. (2009). Moving out of IRF (initiation-response-feedback): A single case analysis. Language Learning, 59(4), 796-824.

Young, R. (2011). Interactional competence in language learning, teaching, and testing. En E. Hinkel (Ed.) Handbook of research in second language teaching and learning, p. 426-443. London: Routledge. 